\title{
Hurricanes: Catastrophic Effects and Their Physical Nature
}

\author{
G. S. Golitsyn \\ A.M. Obukhov Institute of Atmospheric Physics, RAS, Moscow, \\ Russian Federation
}

\section{Introduction}

Hurricanes are one of the most formidable and threatening phenomena on Earth. They are forming over the warm water under combination of specific conditions. Therefore, they are not so often as one could expect: about 80 per annum with noticeable interannual variability. North-West Pacific is their most common place where they are called typhoons. The next place in numbers is North Atlantics, then Indian Ocean with few hurricanes per year.

Meteorological term is tropical cyclones, TC, for short because they are formed in latitude belts $5-25^{\circ}$ North or South of equator. To be called hurricanes or typhoons they must have winds $U$ over $33 \mathrm{~m} / \mathrm{s}$. For $U>20 \mathrm{~m} / \mathrm{s}$ the term is tropical storms.

Very few TC are observed in the Southern Hemisphere and mostly in the Indian Ocean. The are never near equator which suggests importance of the Coriolis force, i.e. the rotation of the planet. $\mathrm{TC}_{\mathrm{s}}$ are seasonal phenomena, their season is from July to November in the Northern Hemisphere, mostly beginning of the autumn. Their tracks have pronounced pole ward component and coming to moderate latitudes they transform into ordinary cyclones. The description of TC from the synoptical point of view is presented well in the classical book by E.Palmen and C.W.Newton "Atmospheric Circulation Systems" [1]. TCs are important elements of the General Circulation of our Atmosphere bringing substantial amount of moisture and angular momentum into the middle latitudes.

TCs are destructive for humans: many ships sink when they happened to be under their action. At their landfall, they destroy much of the property and many people and life perish in general from high winds and massive precipitation often causing floods. Coastal areas are devastated by giant ocean waves. The single TC Katrine in August 2005 devastated New Orleans and many smaller towns in Louisiana, Mississippi and Alabama with overall damage of hundreds billions of US dollars. TC cause most of the damage of hydro meteorological extreme events and the price is increasing during last decades. Now the damage is several times larger than that from earthquakes, volcanoes and other geophysical phenomena. Only one historical TC in Bangladesh in 1975 took about half a million human lives. To diminish the damage the building construction codes have been developed, and should be observed and the warning systems must be in use for population on approaching TC. If similar systems would be in 1975 in Bangladesh the life toll there could be lesser by many times. 
But they also bring large amounts of precipitation, and many Chinese collegues were telling this author that this precipitation is vital for the agriculture in PRC, so the overall effects of TCs into China is positive. Forecast of their formation and evolution is of great importante for many countries, especially for USA, PRC, Japan, Central American states, India, Australia. But to forecast a phenomenon one should understand and model it sufficiently well. However, our understanding is incomplete, especially of the formation stage. TCs are formed in a day or even half day[1].

For the orientation in the physics of a TC one should look wide around onto our atmosphere for something similar. Satellite images of the Earth surface are giving us detailed information about what is going on in the atmosphere. We present on Figs 1, 2 and 3 examples of intense atmospheric vortices, IAV. On Fig.1 we see a typical TC near Florida. Fig. 2 gives us a view of a polar hurricane, polar mesocyclone, of much smaller size than a TC, so it is called polar low, PL, and Fig.3 presents a quasitropical cyclone QTC, over Black Sea. At the fall season such QTCs are occasionally formed over Mediterranean Sea, and very rare over the Black Sea. We need to understand size, energy or winds and conditions for their development.

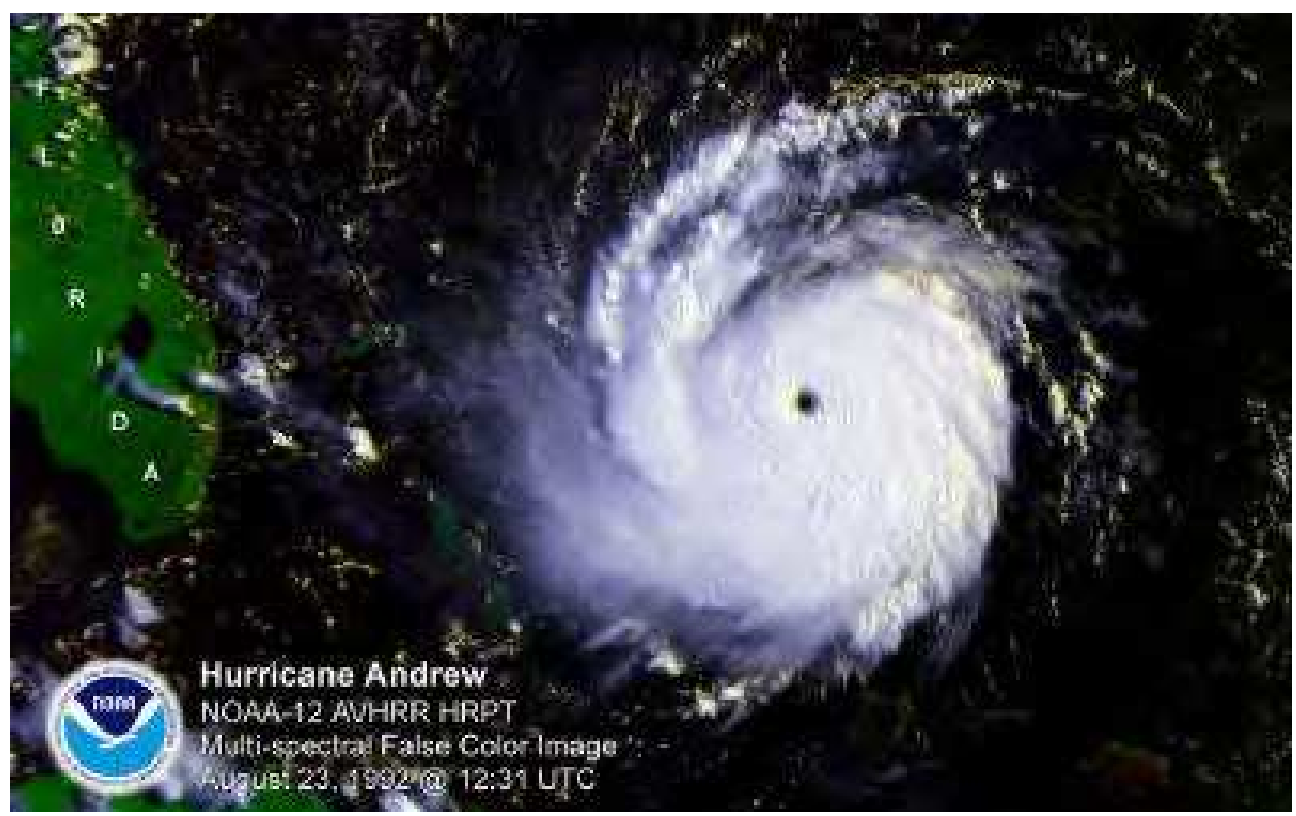

Fig. 1. Typical TC, hurricane Andrew near Florida, 23 August 1992.(The figure captions for paper Hurricanes, by G.S. Golitsyn)

Here we consider conditions for forming intense atmospheric vortices hurricanes and typhoon, polar lows, their physical nature, scales for their winds and sizes, their parameterizations in terms of thermodynamic disequilibrium between ocean and atmosphere. This would allow us to formulate some quantitative necessary conditions for their generation. 


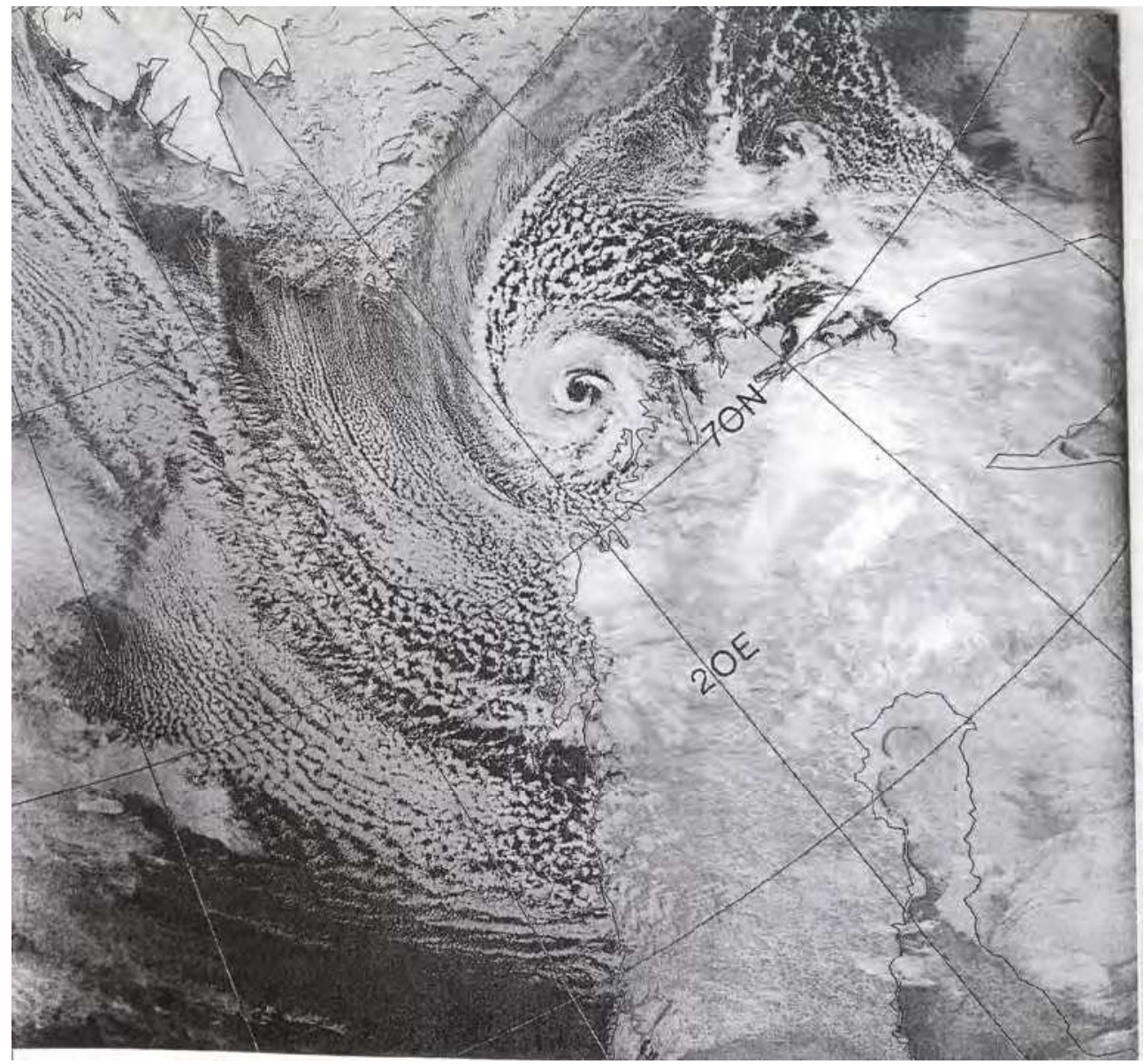

Fig. 2. Polar low, polar mesocyclone to the North of Scandinavia, February 1987. (The figure captions for paper Hurricanes, by G.S. Golitsyn)

Hurricane winds are of $33 \mathrm{~m} / \mathrm{s}$ or larger, vortices with winds between 20 and $33 \mathrm{~m} / \mathrm{s}$ are called tropical storms, and lesser winds are typical for tropical, depressions, de crease in the pressure, or geopotential fields with pressure drops of about $\delta p \sim 10 \mathrm{hPa}$. Initial stage of a TC is a depression but only of order $10 \%$ of such depressions evolve to a TC.

The base for a TC development is a substantial thermodynamic disequilibrium between the ocean surface water and the atmosphere as was visit clearly stated by Riehl [2] in 1950, and Kleinschmidt [3] in 1951.This means that the water must be warmer than the atmospheric air. This is observed during the colder seasons and explains the seasonality of a TC cycle. The very first quantitative condition was found in 1948 by Eric Palmen [4] and it was stating that the temperature of the water must be warmer than $T_{s} \geq 26^{\circ} \mathrm{C}$. 


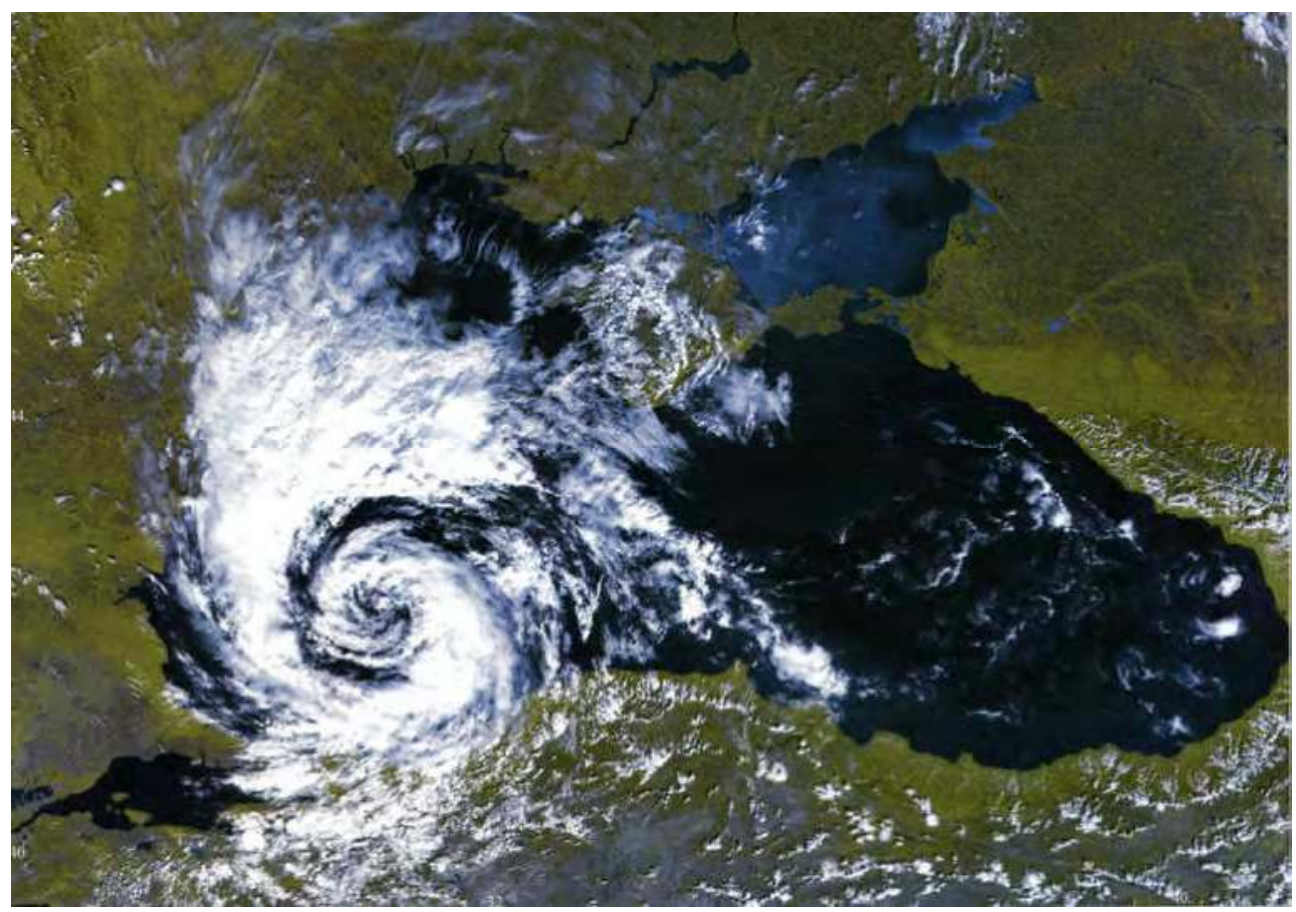

Fig. 3. Quasi-tropical cyclone over the western part of Black Sea, 25 September, 2005. (The figure captions for paper Hurricanes, by G.S. Golitsyn)

The other conditions for such a development were first described by William Gray [5]. They are:

i. The static stability of the atmosphere must be small, i.e. the vertical temperature gradient should be close to the wet adiabatic one;

ii. The tropospheric air about the planetary boundary layer should have enough moisture, otherwise the ascending air being entrained by dry air from above would not condense and the heat of condensation is the main fuel for TCs;

iii. The vertical wind shear should be small, otherwise the hurricane column would be destroyed;

iv. The surface waters should be warmer than the air of lower few meters (usually, the reference height is $10 \mathrm{~m}$ );

v. The upper mixed layer of the ocean (usually it is about 50-100 m) should be thick enough to supply the sufficient energy for a TC;

vi. Ocean temperature, i.e. of UML, should not have large horizontal variation in order to supply the moving TC with necessary fuel.

These conditions should be quantified and that would be a content of the next Sections. 


\section{Physics of the hurricanes and other IAVs}

\subsection{Fluxes between ocean and air}

The main physical process to resolve the thermodynamic disequilibrium in fluids under the gravity field is convection. Convection is an ascent of lighter fluid into a denser one. If the air is windy, as it is always the case, then the air extracts from the water surface the sensible heat and the latent heat as the water vapor and the ocean is losing its heat content or enthalpy. The vapor is condensed at some height because the air temperature is decreasing with height. The heat of condensation is the main source of the energy supply for a TCs. The ascending air causes a drop of pressure at its base and the continuity equation of fluid causes the convergence of adjacent air. Because the Earth is a rotating planet the converging air brings in the convection column its angular momentum, the sum of absolute momentum due to the Earth's rotation and what is of relative vortical motion in the environmental flow. Concentration of the momentum in the ascending convective column enhances the azimuthal velocity in the column. The increased wind causes more evaporation and the sensible heat flux from the water surface which in its turn enhance the wind, etc. The complicated balance between convection strength, winds and energy dissipation, mostly within the boundary layer over water, determines the energy of a TC. Its vertical extent is up to tropopause, i.e. $15-18 \mathrm{~km}$ in the tropics. $\mathrm{TC}_{\mathrm{s}}$ do not propagate further up because of the very strong static stability of the stratosphere. The acquired angular momentum is dispersed at the top of a TC in the form of anticyclonic spirals so well seen by satellites.

This qualitative picture was quantisized to some extent by Kerry Emanuel and was called by him as WISHE (Wind Intensification by Sensible Heat and Evaporation). The detailed description of this one can find in his two review papers [6, 7]. At the end of the latest review he concludes that the origin of TCs was still unclear and that it might be not just one way for their origin. To resolve the problem one needs more observations and their detailed analysis and numerical experiments with a kilometer resolution, not available in the early 2000 s for the domains of a few thousand kilometers.

Let us consider hurricanes, TC and PL, as convection vortices in rotating fluids. The theory and extensive experimentation can be found in the book [8] and research papers [9-12]. The first results are for the scales of velocity and area [9-14] were obtained from dimensional analysis. For convection the main governing parameter is the buoyancy flux, which in a steady state is equal to the rate of the kinetic energy dissipation [15] it the Boussinesq approximation:

$$
b=\varepsilon=-\frac{g}{\rho}<\rho^{\prime} w^{\prime}>=-\frac{g}{p T}<w^{\prime} T^{\prime}>-0.622<w^{\prime} g^{\prime}>
$$

where $g$ is the gravity acceleration, $\rho$ the gas density, $w$ prime signs mean fluctuations relative to mean state the vertical velocity component, $T$ the gas temperature, $g$ the water vapour mixing ratio, angular brackets mean statistical averaging

The moist gas thermodynamics is assumed $[6,7]$ here when the density of an air parcel is here equal to the sum of air and vapour densities: 


$$
\rho=\rho_{a}+\rho_{w}=\frac{p_{a}}{R_{a} T}+\frac{e_{s}}{R_{w} T}=\frac{p_{a}}{R_{a} T}\left(1+\frac{\mu_{w}}{\mu_{a}} \cdot \frac{e_{s}}{p_{a}}\right) \cong \frac{p_{a}}{R_{a} T}\left(1+0.622 \frac{e_{s}}{\rho_{a}}\right),
$$

where the ideal gas equation of state is assumed, $R_{a}=R / \mu_{a}{ }^{\prime} R_{w}=R / \mu_{w}$, constants for a gas with molecular weight $\mu, e_{s}$ is the saturation water vapour pressure whose dependence on the temperature is described by the Clapeiron-Clausius equation. The mixing ratio is related to the specific humidity $e(T)$ as

$$
q=\frac{\mu_{w}}{\mu_{a}} \frac{e}{p}=0.622 \frac{e}{p}
$$

where $\mu_{\mathrm{w}}=18.015$ and $\mu_{\mathrm{a}}=28.97$ are corresponding molecular weights for water vapour and air, $p$ is the atmospheric pressure. The decrease of the air density, as compared with the dry air at the same pressure, is equal to

$$
\Delta \rho=-\frac{0.622}{R_{a} T} p \Delta q=-\frac{0.378}{R_{a} T}, \quad 0.378=\frac{\mu_{a}-\mu_{w}}{\mu_{a}},
$$

The thermodynamic disequilibrium between the ocean and the atmosphere is resolved in the process of the two media interaction by means of the momentum, heat and moisture fluxes. The study of these fluxes, especially at high winds, is still one of the main goals of the physical oceanography, complications are due to presence of the waves at the water surface.

The fluxes of the momentum, sensible and latent heat are described by the so-called bulk formulae [16-19]:

$$
\begin{gathered}
\tau=\rho\left\langle w^{\prime} u^{\prime}\right\rangle=c_{D} \rho U^{2}=\rho u_{*}^{2}, \\
F_{S H}=\rho c_{p}\left\langle w^{\prime} T^{\prime}\right\rangle=c_{T} c_{p} \rho U \Delta T, \\
F_{L H}=\rho\left\langle g^{\prime} w^{\prime}\right\rangle=c_{E} \rho U \Delta q,
\end{gathered}
$$

where $\mathrm{U}$ is the wind velocity at the reference level $10 \mathrm{~m}, c_{D}, c_{T}, c_{E}$ are the drag coefficients for the momentum, sensible heat and moisture, $\Delta T=T_{s}-T_{a}$ is the temperature difference between sea surface and the air at $10 \mathrm{~m}, \Delta q$ is the difference between the mixing ratios of the water vapour at the sea surface where $T=T_{s}$, and the relative humidity is $100 \%$, and the one at $\mathrm{T}(10 \mathrm{~m})$. According to ocean climatology [20] the relative air humidity $r=q(T) / q_{s}(T)$ over all oceans at all latitudes in all seasons is close to $80 \%$, i.e. $r \approx 0.8$. It decreases to 0.7 only occasionally. Emanuel [6] in his review used $r \approx 0.75$. In this case the Bowen number the ratio of the sensible and latent heat fluxes, would be smaller by a factor $\left(1-r_{2}\right) /\left(1-r_{1}\right)=0.25 / 0.20=1.25$.

The formulae (4)-(6) follow from similarity considerations and are obtained by the analogy with a technical problem of a plate cooled by a colder air flow with velocity $U$. The 
coefficient $\mathrm{c}_{\mathrm{T}}$ in such a problem is known as the Stanton number [15]. It should be noted that under the modern instrumental precision [19] $c_{T} \approx c_{E}$ in (5) and (6), i.e. these coefficients are assumed further to be equal.

\subsection{Buoyancy flux}

The buoyancy flux is described by eq.(1). Using the bulk formulae for sensible and latent heat, eqs. (5) and (6), we obtain from eq.(1).

$$
b=-c_{T} \frac{\Delta T}{T} g U\left(1+0.622 \frac{c_{E}}{c_{T}} T \frac{\Delta q}{\Delta T}\right) .
$$

Remembering that $c_{T}=c_{E}$, according to [17-19], and taking into account eqs.(3) and (4) we re-write eq.(8) as

$$
b=-g^{\prime} U, \quad g^{\prime}=c_{T} \frac{\Delta T}{T} g\left(1+0.378 \frac{\Delta e}{p \Delta T}\right), \quad \Delta e=e_{s}\left(T_{s}\right)-r e_{s}\left(T_{a}\right),
$$

where $g^{\prime}$ may be called the reduced gravity acceleration. Following [20] the saturated water vapour pressure as a function of temperature can be conveniently presented (in $\mathrm{Pa}$ units) as

$$
\begin{aligned}
& e_{s}(T)=\exp \left[23.7812-4157(T-33.91)^{-1}\right] \\
& e_{a}(T)=\exp \left[23.8014-4157(T-33.91)^{-1}\right]
\end{aligned}
$$

These expressions were obtained from the integration of the Clapeyron-Clausius equation by some approximations and are valid with the accuracy of a few hundredths of a percent in all practical ranges of atmospheric temperatures. One can find other formulae for the air humidity, but we use these ones in the calculations below. The difference in the first term in the square brackets in eqs.(10) and (11) is related to the fact that for water with salinity $34 \%$ the pressure of the saturated water vapour is $98 \%$ of the pressure over distilled water [18].

The first formula (9) shows that the buoyancy flux is the product of the reduced gravity acceleration and the wind velocity. It has the dimension of power per unit mass. In statistically stationary conditions, i.e. in a steady state, this flux is, evidently, equal to the rate of generation and dissipation of the convective kinetic energy also per unit mass. This follows with the convective energy balance equation [15]. This does not hold in nonstationary conditions, e.g. the buoyancy flux in penetrative convection (see below section 4) decreases linearly with height, expending its energy to the lift and warm the air [2123].

Equation [9] determines the buoyancy flux over the water surface in the absence of condensation. Observations show that for TC, as well as for PL, the condensation starts forming clouds at levels of a few hundred meters (300-400 $\mathrm{m}$ ) above the ocean surface. In TC 
the clouds rise up to the tropical tropopause of 15-18 km. In PL the cloud tops reach 5-6 km [24]. In this case there should not be a substantial error if we neglect the relatively thin subcloud layer and assume that the latent heat of condensation starts to be felt right at the water surface. In any case, the enthalpy flux leaving the water is just the sum of sensible and latent heats fluxes in the air. We can now rewrite eqs.(9) as

$$
b=-c_{T} \frac{\Delta T}{T} g\left(1+\mathrm{Bo}^{-1}\right)=g^{\prime} U, g^{\prime}=c_{T} \frac{\Delta T}{T} g\left(1+\mathrm{Bo}^{-1}\right) .
$$

We introduce here the Bowen number, $\mathrm{B}_{\mathrm{O}}$ the ratio of the sensible and latent heat fluxes. Its inverse ratio is equal to

$$
\mathrm{Bo}^{-1}=\frac{\mu_{w}}{\mu_{a}} \frac{L \Delta e}{p c_{p} \Delta T}=0.610 L_{1} \frac{\Delta e}{\Delta T}
$$

where $L=10^{6} L_{1}$, is the latent heat of evaporation, $p=p_{s}=1.013 \cdot 10^{5} \mathrm{~Pa}$ is the globally mean air pressure at the sea level. According to [20] the latent heat in SI units is a function of the absolute temperature in the following form

$$
L=1.91846 \cdot 10^{6}\left[T(T-33.91)^{-1}\right]^{2} .
$$

In the observed range of temperatures of the ocean's open surface from $-2^{\circ} \mathrm{C}$ to $+31^{\circ} \mathrm{C}$ the value of $L_{1}=10^{-6} \mathrm{~L}$ changes from 2.506 to 2.430 or by $3 \%$. This should be taken into account in exact computations.

Note that eqs.(8) and (9) show that buoyancy flux exists even at $\Delta T=0$, i.e. when both temperatures of air and water are equal. The flux then is only due to the lighter water vapour as compared to the unsaturated air. The last flux vanishes when the air is warmer than the water, as it follows from eq.(9) by

$$
\Delta T=T_{a}-T_{s}=0.378 \frac{\Delta e}{p}
$$

where from in the tropics at $\mathrm{T}=300 \mathrm{~K}$, or $27^{\circ} \mathrm{C}$, and $\frac{\Delta e}{p} \approx 10^{-2}$, we get $\Delta \mathrm{T} \approx 1 \mathrm{~K}$.

The fluxes of the sensible and latent heat, and therefore the buoyancy flux, depend on the wind speed $U$, see the bulk formulae and eqs.(9) and (12).

The convective vortices are processing the ambient air by rising it up. These processes create high winds by concentrating the ambient angular momentum, which intensifies the convective vortices increasing the fluxes in its turn. The process is stabilized when the dissipation of the kinetic energy proportional to the cube of the wind and occurring mainly in the boundary layer [24] becomes equal to the rate of the energy generation [13, 14, 24]. These are highly non-linear processes but fortunately the situation can be described by invoking parameterizations depending on fluxes in consideration. 


\section{Scaling for steady vortices}

\subsection{Convection in rotating fluids}

The average lifetime of TC is about a week, though one such vortex is known to exists for a month [25, 26]. A PL lives of order a day or even less [27]. The theory of convection for rotating fluids assumes the steady state and constant fluxes [8-10]. Therefore the theory gives the right scales for the winds and size at a steady state but even for the development stage it should not be much in error.

The main parameters determining the convection should be the buoyancy flux $b$ with $[b]=\mathrm{L}^{2} \mathrm{~T}^{-3}$, where after J.C.Maxwell square bracket mean the dimension, $\mathrm{L}$ and $\mathrm{T}$ are dimensions of length and time, and the Coriolis parameter

$$
l_{c}=2 \omega \sin \Theta,
$$

where $\omega$ is the rotation rate and $\Theta$ is the latitude. Also an external length scale is present, e.g. the planetary radius R. From these three parameters determine a non-dimensional similarity criterium [9] which we take in the form

$$
\text { Ro }=b^{1 / 2} l_{c}^{-3 / 2} R^{-1}
$$

which happened to be the Rossby number [9] as we would see just in few lines. When Ro $<<1$ the rotation is felt strongly. Now we suppose that the characteristic vortex sizes $d$ are much less than R. From the two remaining parameters, $b$ and $l_{c}$, we construct the scales of length $d$, say, diameter of a TC, and velocity:

$$
\begin{aligned}
& d=c_{1} b^{1 / 2} l_{c}^{-3 / 2} \\
& U=c_{2} b^{1 / 2} l_{c}^{-1 / 2}
\end{aligned}
$$

where $c_{1}$ and $c_{2}$ are numerical constants determined experimentally [10-12]. It was found that $c_{1} \approx 10$ and $c_{2}=1.7 \pm 0.1$ so $c_{2}^{2} \approx 3$ which we shall us later.

After combining eqs.(12) and (19) we obtain a relationship between the velocity and reduced gravity:

$$
U \approx 3 g^{\prime} l_{c}^{-1}
$$

and the reduced gravity $g^{\prime}$ is determined by the second eqs.(12). The relationship (20) expresses the wind velocity in a hurricane through the parameters of the thermodynamic disequilibrium between the atmosphere and ocean.

\subsection{Differences between TCs and PLs}

The eqs.(18) explains why a PL is typically three times smaller than a TC [27]: at $\Theta=20^{\circ}$ we have the Coriolis parameter (16) equal to $0.5 \cdot 10^{-4} \mathrm{~s}^{-1}$, but for $\Theta=70^{\circ}$, as on Fig.2, $l_{c}=1.37 \cdot 10^{-4} s^{-1}$.According to (18), if the other conditions equal the diameters should be 
different by $\left[l_{c}\left(70^{\circ}\right) / l_{s}\left(20^{\circ}\right)\right]^{\frac{3}{2}}=(1.37 / 0.5)^{\frac{3}{2}}=4.5$ times. As we will see later the reduced gravity at high latitudes is about twice of that in the tropics due to much higher static stability of the polar atmosphere than in the tropics and much lower Bowen ratio. Therefore to reach the hurricane winds of $33 \mathrm{~m} / \mathrm{s}$ the reduced gravity is about twice or more than in the tropics. This reduces the size difference for TC and PL to about three times as it is in reality.

The enthalpy fluxes from the ocean to the atmosphere are of order $700 \mathrm{Wm}^{-2}$ or more at hurricane winds of $\mathrm{TC}$, and for PL they may reach $1.5-2 \mathrm{kWm}^{-2}$ or even more. Such high fluxes were measured in winter near Newfoundland and east of Japan (private communications by Yu.A.Volkov and S.S.Lappo, early 1990s).In [13,14] one can find special tables for both tropical and polar conditions for the reduced gravity, Bowen ratio, enthalpy fluxes and corresponding velocities $U \geq 33 \mathrm{~m} / \mathrm{s}$ or higher at the ranges of polar temperatures at the sea surface of 0 to $8^{\circ} \mathrm{C}, \Delta \mathrm{T}=20 \mathrm{~K}$, and for tropical range $\mathrm{T}_{\mathrm{s}}$ from 24 to $30^{\circ} \mathrm{C}, \Delta \mathrm{T}=2 \mathrm{~K}$ and $1 \mathrm{~K}$. As we noted above the air relative humidity plays important role, especially in the tropics. If we decrease there $\mathrm{r}$ from $80 \%$ to $70^{\circ}$ the Bowen ratio would increase in 1.5 times. Such an increase may happen in Easterly waves in Atlantics bringing dry air from Sahara [1].

The high IAV winds not only take away the heat content from the upper mixed layer, UML, of the ocean but stir it additionally. If $\mathrm{UML}$ is $\mathrm{H}=50 \mathrm{~m}$ deep with the (flux) out of it $\mathrm{F}=1 \mathrm{kWm}^{-}$ 2 during one day, $t_{1}$, it would cool by $0.43 \mathrm{~K}$.

Quite often few degree of cooling of UML is observed after a TC passage which means that this layer is mixed down to the thermocline by high surface waves breaking bringing up colder deep waters. This means that good TC, and PL, prediction models must have a deep enough block for the ocean description and also for the wind surface a waves. The colder surface waters would decrease $\Delta T$, the surface air temperature difference, diminish the enthalpy fluxes and weaken the vital feedback for IAVs.

\section{Atmospheric stratification and penetrative convection}

From the point of view by Gray [4] the most important condition for a TC origin is the static stability of the atmosphere. It is determined by the vertical air density gradient

$$
\Gamma=\left(\frac{d \rho}{d z}\right)_{a}-\frac{d \rho}{d z},\left(\frac{d \rho}{d z}\right)_{a}=\Gamma_{a}=-\frac{\rho g}{c_{a}^{2}}, c_{a}^{2}=\chi R_{a} T,
$$

where $c_{a}$ is the adiabatic sound velocity in an ideal gas, $\chi=\frac{c_{p}}{c_{v}}$ is the adiabatic exponent. The hydrostatic equation $d p=-\rho g d z$ is used here. A convenient measure of the density gradient is the square of the Brunt-Väisälä frequency $N^{2}=-\left(\frac{g}{\rho}\right) \Gamma$. In the Boussinesq approximation, it is equal to 


$$
N^{2}=-\frac{g}{T}\left(\frac{d T}{d z}-\gamma_{a}\right), \quad \gamma_{a}=-\frac{g}{c_{p}}=-9.8 \frac{\mathrm{K}}{\mathrm{km}}
$$

When a source of buoyancy at the lower boundary of a stably stratified fluid layer starts to act, then a convective boundary layer develops. Its height increases with time $t$ as [21-23]:

$$
h(t)=(2 b t)^{\frac{1}{2}} N^{-1}
$$

This expression should also take into account the entrainment from above of the ambient air which would increase r.h.s. of eq.(23) by some $20 \%[22,23]$. The typical values of $\Gamma=\frac{d T}{d Z}$ is about $-6 \mathrm{~K} / \mathrm{km}$. It is about $-6.5 \mathrm{~K} / \mathrm{km}$ at low latitudes and $-5.5 \mathrm{~K} / \mathrm{km}$ at high latitudes [28]. In the first case $N \approx 1 \cdot 10^{-2} \mathrm{~s}^{-1}$, and in the second $N=1.24 \cdot 10^{-2} \mathrm{~s}^{-1}$. Higher internal wave frequency means that penetration of convective patterns at high latitudes is some $25 \%$ slower than at low latitudes.

We know time of development for both TC and PL. The height of these $\mathrm{IAV}_{\mathrm{s}}$ is correspondingly $15 \div 18 \mathrm{~km}$ and $5 \div 6 \mathrm{~km}$. Accepting these two conditions we may estimate what kind of thermodynamic disequilibrium is necessary to reach the observed tops of the vortices in the prescribed time, using (23):

$$
b=\frac{N^{2} h^{2}(t)}{2 t}
$$

with the buoyancy flux expressed by eq.(12) and the wind speed by eq.(20). From the eqs.(24), or (23), we have

$$
\frac{c_{T} g}{h N}\left(\frac{2 t}{l_{c}}\right)^{\frac{1}{2}} \frac{\Delta T}{T}\left(1+\mathrm{Bo}^{-1}\right)=A \frac{\Delta T}{T}\left(1+\mathrm{Bo}^{-1}\right), \quad A=\frac{c_{T} g}{h N}\left(\frac{2 t}{l_{c}}\right)^{\frac{1}{2}}
$$

where A does not depend on the disequilibrium conditions.

If we take $N \approx 1 \cdot 10^{-2} \mathrm{~s}^{-1}$ and typical value $b=0.03 \mathrm{~m}^{2} \mathrm{~s}^{-3}$, then the time of reaching $18 \mathrm{~km}$ by convection would be several days. This clearly illustrates the importance of the small static stability. We assume $N=3 \cdot 10^{-3} \mathrm{~s}^{-1}$ which corresponds to the difference of only about $0.3 \mathrm{~K} / \mathrm{km}$ between the actual and the dry adiabatic temperature gradient while normally the difference is $(9.8-6.5)=3.3 \mathrm{~K} / \mathrm{km}$. Fig.4a,b presents the non-dimensional criterion (25) on the plane $\left(\Delta T, T_{s}\right)$. The curves with number larger or equal to 1 (thick curve for the last case cover the area of the parameter space with a possibility of a TC development at $20^{\circ} \mathrm{N}$ in 11 hours or $4 \cdot 10^{4} \mathrm{~s}$. Due to the independence of the multiplier $\mathrm{A}$ in (25) on either $\Delta \mathrm{T}$ or $\mathrm{T}_{\mathrm{s}}$ this graph has a wide interpretation. E.g. the curve labeled 0.8 can become the thick curve labeled 1 if we enlarge A by a factor 1.25 by increasing time by $1.252=1.5625$, or decreasing $\mathrm{N}$ by a factor 1.25 . 
$r=0.8$

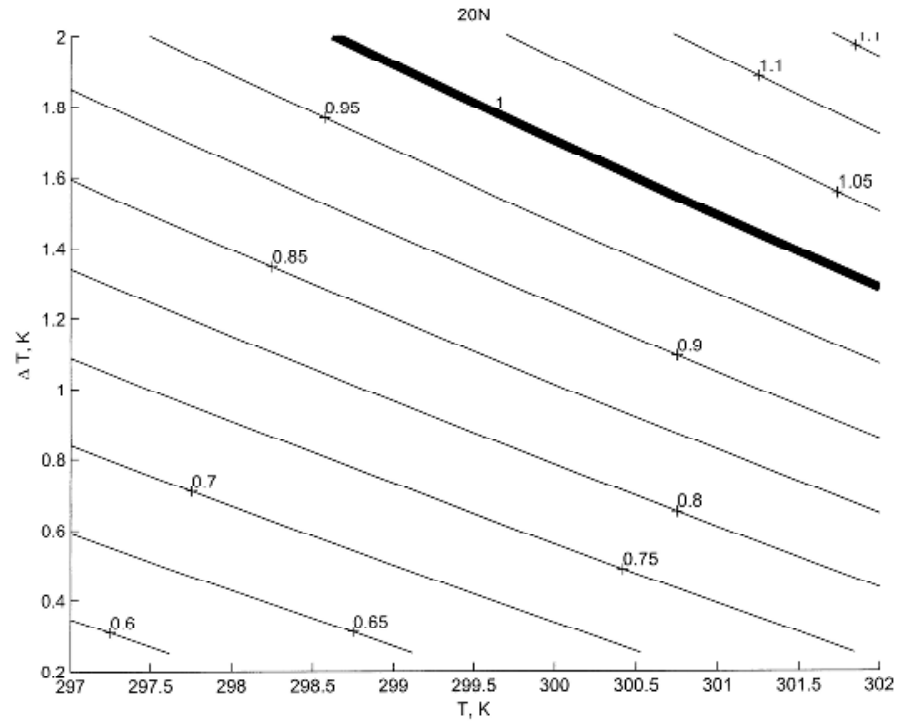

a)

$r=0.7$

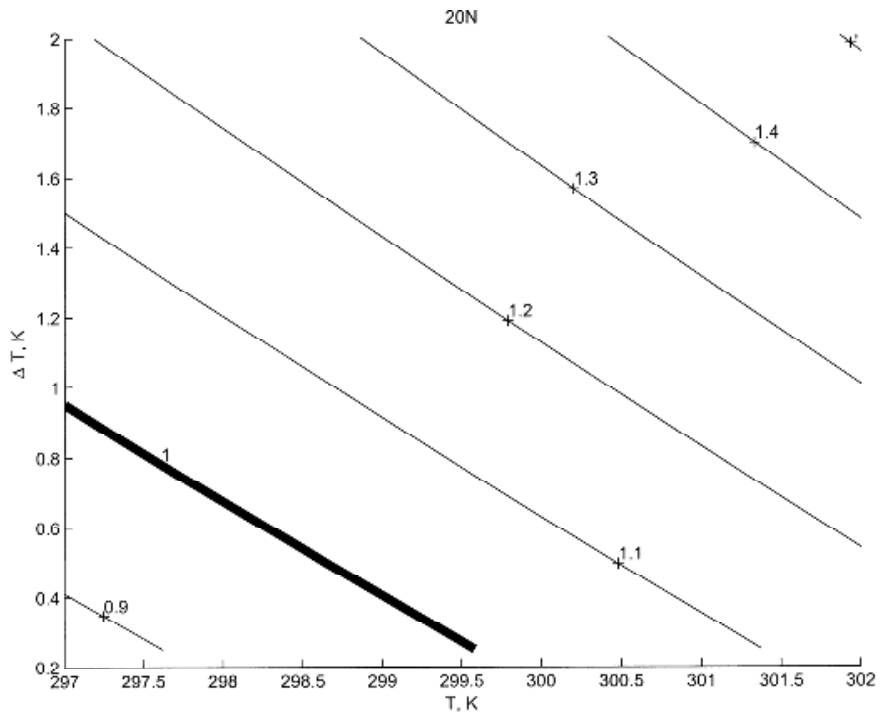

b)

Fig. 4. Diagram for tropical cyclone, TC origin conditions depending on sea surface temperature $\mathrm{T}_{\mathrm{s}}$ and the difference $\Delta \mathrm{T}=\mathrm{T}_{\mathrm{s}}-\mathrm{T}_{\mathrm{a}}$, where $\mathrm{T}_{\mathrm{a}}$ is the atmospheric temperature at 
At $z=10 \mathrm{~m}$ reference height $\mathrm{z}=10 \mathrm{~m}$. a) For relative humidity $\mathrm{r}=0.8$ and $\mathrm{b}$ ) for $\mathrm{r}=0.7$. The thick line 1 is the critical curve where development of a TC becomes possible in the parameter space to the right above this curve. (The figure captions for paper Hurricanes, by G.S. Golitsyn)

Figs.4a and $4 \mathrm{~b}$ differ only by the values of the relative humidity $r$ of the atmosphere. Changing $r$ at Fig.4a from 0.8 to $r=0.7$ at Fig.4b increases the Bowen ration by a factor of 1.5. Only this replacement decreases the necessary temperature difference $\Delta T$ to a fraction of a degree, still presenting a possibility for TC formation at high SST.

At Fig.5 we present similar results for polar lows or $\mathrm{PL}_{\mathrm{s}}$, at $\Theta=70^{\circ} \mathrm{N}$ and $\Theta=70^{\circ} \mathrm{N}$, $N=1 \cdot 10^{-2} \mathrm{~s}^{-1} \quad t=2 \cdot 10^{4} \mathrm{~s}=5.5 \mathrm{~h}$. Here the necessary temperature difference is about $25 \mathrm{~K}$, or larger. This is an agreement with results of simulation of PL s[27] requiring $\Delta T>20 \mathrm{~K}$ in order to form such vortices. The same kind of reinterpretation, as for TC case, can be made here with curves labeled with different numbers around 1. One should also note that similar conditions might be also applicable to VHT, vertical hot tower formation.

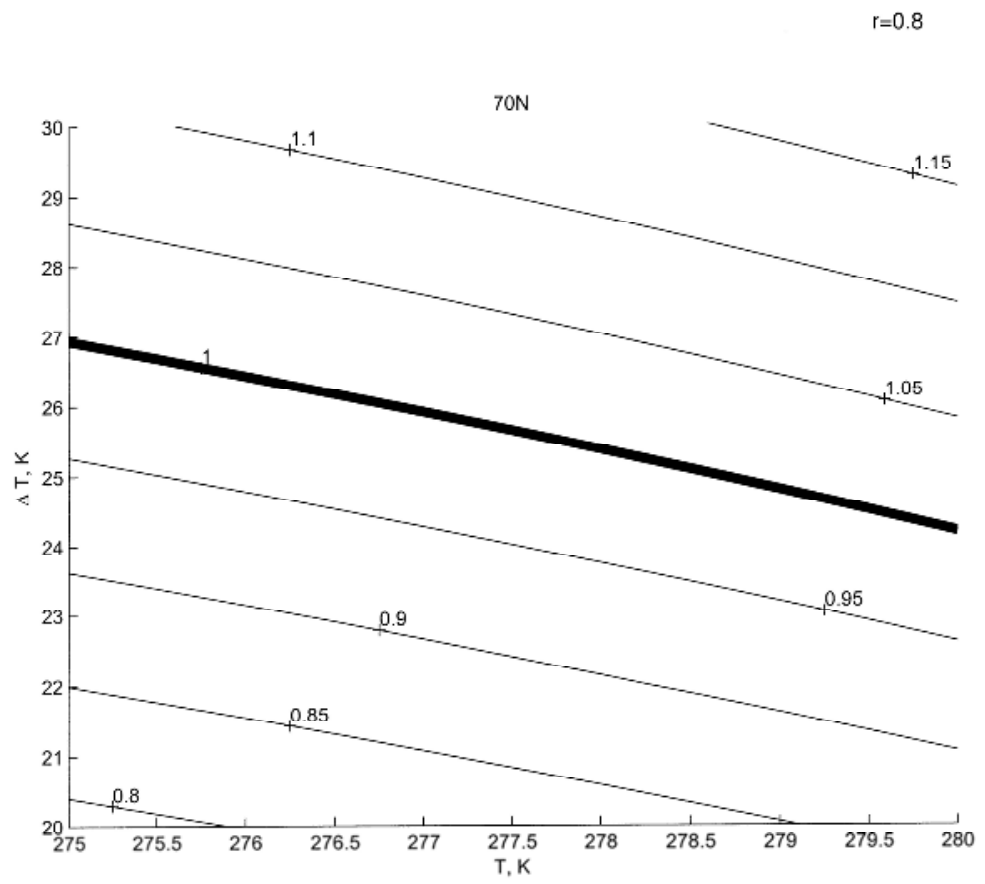

Fig. 5. Same as at Fig.4.but for the origin of a polar low, PL, at $\mathrm{r}=0.8$ (The figure captions for paper Hurricanes, by G.S. Golitsyn) 
In $[13,14]$ there are also velocity, estimates for IAVs using the boundary layer energy balance [24] which is independent from Coriolis parameter. These estimates for conditions used for construction of our Fig. 4 and 5 give results within $20 \%$ of the presented above on these figures. However, the last estimates require more ad hoc parameters and therefore suffer more uncertainties.

\section{Problems associated with IAVs origin}

The potential area should be large enough or at least greater than the scale of a generated vortex $d$ presented by eq.(18). The necessary homogeneity of the water surface temperature leads to a condition

$$
\left(d T_{s} / d x\right) d<<\Delta T=T_{s}-T_{a}
$$

where $x$ is the horizontal coordinate.

The condition of the small vertical wind shear $[4,5]$ can be written as

$$
t_{d v p} \Delta U<<d,
$$

where $t_{d v p}$ is the time of the vortex development and $\Delta U=(d U / d z) h$ is the ambient wind velocity difference between the lower and upper parts of the developing vortex. These two conditions are enough for the energy supply to feed the development of the vortex, which should not be sheared apart.

Emanuel [7] noted in his review that there could be several mechanisms for the origin of a TC. The observations show that TC is always originating from a tropical depression, TD. But only a small part, about one tenth, of them may develop a hurricane. $\mathrm{TD}_{\mathrm{s}}$ are accompanied by developing vortical hot towers, VHT, reaching the tropopause and 20 to $30 \mathrm{~km}$ in diameters.

The analysis of satellite images of $\mathrm{TD}_{\mathrm{s}}$ preceding the formation of a $\mathrm{TC}$, shows that normally there are about 20-30 VHT in a TD. But to develop a TC there should be more VHTs, about $30 \div 40$ as revealed the analysis in 1980s at the Colorado State University. In the extensive series of computations [30-32] it was demonstrated how VHTs develop, acquire initial vorticity, and merge by bringing in this way the planetary angular momentum of the ambient air from thousands of kilometers away. In [14] one can find an analysis of vorticity balance at merging $40 \mathrm{VHTs}$ with their initial azimuthal velocities of only $0.5 \mathrm{~m} / \mathrm{s}$ may produce a TC with the maximum velocity of $40 \mathrm{~m} / \mathrm{s}$ at the distance of $50 \mathrm{~km}$ from the center of the TC. There is a review [14] of theoretical works in hydrodynamics on the process of merging vortices of the same sign.

The presence of an initial vortex [4,5], or forming it at an initial stage of a TC generation is a very important condition. For instance the cyclone over Black Sea (Fig.2) at the end of September 2005, successfully modeled in [34] by a MM5 model, could not be formed without such a vortex brought in by Balkan cold air. Such a necessity was also clearly demonstrated by a series of the 3D high resolution computations initiated by Montgomery 
(see [32] and references therein). It was shown there that the full extent of the wind-induced surface heat exchange follows the stage of the initial vortex formation which could start even at the nominal trade wind velocities of order $10 \mathrm{~m} / \mathrm{s}$. This vortex is intensified by locally buoyant VHTs and near surface convergence induced by them within the boundary layer.

A number of numerical studies [33-35] reveal that a TC may be formed from a random convection patterns without systematic initial winds if the integration is long enough, $5 \div 15$ days. Most of these computations assume initial temperatures and its and humidity profiles from [36] where $T_{s}=28^{\circ} \mathrm{C}$. It is due to cooperative interaction between large-scale moisture in the troposphere, locally enhanced sea-surface fluxes leading to aggregation of convection [33], in other words merging of VHTs.

There is a hope that during nearest year's extensive high-resolution computations together with specially organized field campaigns would clarify the ways for TCs formation, possibly not just one way.

We see that TCs may be formed at temperature difference of a fraction of a degree and small static stability of the atmosphere, for which we should know rather precisely the temperature and humidity profiles in the boundary lager. All these is unattainable with the present satellite instrumentation and data retrieval techniques. This suggests that operational forecast of place and time of a TC origin is a matter of a distant future, if possible at all. What present weather forecasting operational models can perform that to follow the track and intensity evolution of a TC. The precision of the performance is improving but its further improvement is highly desirable. With $\mathrm{PL}_{\mathrm{s}}$ the situation is better: when cold polar air masses intrude over open water and temperature difference between air and water surface is $20-40 \mathrm{~K}$ the disequilibrium is striking and forecasts are easier.

Importante question is what would happen to TCs in a warmer climate. This is one of the most important areas for IPCC kind of studies. There is a large number of papers already, starting with [37] analyzing empirical evidence. It is concluded that if the number of $\mathrm{TC}_{s}$ may not increase but their destructiveness proportional to $\mathrm{U}^{3}$ is increased during the last 30 years when signs of global warning became obvious. There are several modeling studies, e.g. [38, 39] but model result are still inconclusive for a global number of TCs, especially for various basins: Atlantics, Pacific, etc. Though the areas with $T_{3} \geq 26^{\circ} \mathrm{C}$ are greatly increasing the other conditions essential for a TC formation may change. At the moment a general result of [37] may be valid but one should wait for more conclusive results in the $5^{\text {th }}$ IPCC Report in 2014.

\section{Conclusions}

The intense atmospheric vortices considered here evolve from the thermodynamic disequilibrium between ocean and atmosphere, therefore their global monitoring could be done by satellites. The sizes of these vortices decrease in general to higher latitudes due to the earth rotation.

The static stability of the atmosphere also plays a role, increasing with latitudes. Some necessary conditions for their generation are described here revealing that the present 
remote sensing systems are not able to present necessary precision for determining the time a place for formation, especially for a TC. Therefore the observation of construction codes and warning systems must be in operation to reduce greatly the damage.

Global warning poses an important question on the evolution and behavior of these vortices in future: would their number increase together with their destructiveness? The present understanding, before the IPCC5 report in 2014, is that the number might still be under one hundred but the winds in them could be higher. Therefore the numerical modeling with higher than $1 \mathrm{~km}$ resolution is required. At present the improvement is urgent of weather prediction operational models in following the trajectories and evolution of these vortices.

\section{References}

[1] Palmen E. and C.W.Newton.1969, Atmospheric Circulation Systems. Academic Press, N.Y. and London. 603 pp.

[2] Riehl, Y.1950. A model for hurricane formation. J.Appl.Phys., 21, 917-925.

[3] Kleinschmidt, E.1951.Gründlagen einer Theorie des tropischen Ziklonen. Arch. Meteorolog.Geophys.Bioklimatol. 4A, 53-71.

[4] Gray W.M., 1968.Global view of the origin of tropical disturbances and storms. Mon.Wea.Rev. 96, 669-700.

[5] Palmen E. 1948. On the formation and structure of tropical cyclones. Geophysics, 3, 2638.

[6] Emanuel, K., 1991. The theory of hurricanes. Annu.Rev.Fluid Mech. 23,175-196.

[7] Emanuel, K., 2003. Tropical cyclones.Annu.Rev.Earth Planet. Sci. 31,75-104.

[8] Boubnov, B.M., and G.S.Golitsyn, 1995. Convection in Rotating Fluids. Dordrecht, Kluwer, 232 pp.

[9] Golitsyn, G.S., 1980. Geostrophic convection. Proc. (Doklady), USSR Ac.Sci. 251 (6), 13561359.

[10] Boubnov, B.M., and G.S.Golitsyn, 1990.Temperature and velocity field regimes of convective motions in a rotating fluid layer.J.Fluid Mech., 219, 215-239.

[11] Fernando, J.H.S., Boyer, and R.Chen, 1991. Effects of rotation on convective turbulence. J.Fluid Mech., 228, 513-547.

[12] Maxworthy, T., and S.Narimosa, 1994. Unsteady turbulent convection into a homogeneous rotating fluid with oceanographic applications J.Phys.Oceanogr. 24, 865-867.

[13] Golitsyn, G.S., 2008.Hurricanes, tropical and polar ones, their energy and size, quantitative criterium of their origin. Izv-Atmos.Ocean Physics, 44 (5), 579 - 590.

[14] Golitsyn, G.S., 2009. Tropical cyclones and polar lows: velocity, size and energy scales, and relation to the $26^{\circ} \mathrm{C}$ cyclone origin criteria. Adv.Atmos.Sci., 26(3), 585-598.

[15] Monin, A.S., and A.M. Yaglom.1975. Statistical Hydromechanics.V.1. MIT Press, 708 pp.

[16] Kitaigorodskii, S.A., 1973. The Physics of the Air-Sea Interaction. Jerusalem, 237 pp.

[17] Smith, S.D., 1989.Evaporation fluxes over sea: An overview. Bound. Layer Meteorol., 47, 277-293.

[18] Kraus, E.B., and J.A.Businger, 1994. Atmosphere-Ocean Interaction.2nd,ed. Oxford Univ.Press, $362 \mathrm{pp}$. 
[19] Fairall, C.W., E.F.Bradley, J.Y.Hare, A.A.Grachev, and J.B.Edson.2003. Bulk parametrizations of air-sea fluxes: Updates and verification for the COARE algorithm. J.Climate, 16(4), 572-591.

[20] Henderson-Sellers, B., 1984.A new formula for the latent heat of evaporation as a function of temperature. Quart.J.Roy. Meteorolog.Soc., 110, 1186-1190.

[21] Turner, J., 1973.Buoyancy Effects in Fluids. Cambridge Univ.Press,367 pp.

[22] Zilitinkevich, S.S., 1987.Theoretical model for the turbulent penetrative convection. Izvestia-Atmos.Ocean Physics, 23(6), 593-610.

[23] Zilitinkevich, S.S. Turbulent Penetrative Convection. Avebury Technical, Brookfield USA, Hong Kong, Singapure, Sydney, 179pp.

[24] Bister, M., and K. Emanuel, 1998. Dissipative heating and hurricane intensity. Meteorol. Atmos.Phys., 65, 223-240.

[25] Riehl, Y., 1954. Tropical Meteorology. Academic Press. N.4. 366 pp.

[26] Golitsyn, G.S., P.F. Demchenko, I.I. Mokhov, and S.G. Priputnev, 1999. Tropical cyclones: Statistical properties of distributions in dependence on intensity and duration. Proc.(Doklady), Russ.Ac.Sci., Earth Sci.Sect., 366 (4), 537-542.

[27] Rasmussen, E.A., and J. Turner, 2003. Polar lows. Mesoscale Weather Systems in Polar Regions, Cambridge Univ.Press, 612 pp.

[28] Mokhov, I.I., and M.G. Akperov, 2006. Verical temperature gradient in the troposphere and its connection to the surface temperature after reanalysis data. Izv.-Atmos.Ocean Phys. 42(4), 467-475.

[29] Hendricks, E.A., M.T. Montgomery, and C.A. Davis, 2004. Role of vortical hot towers in the formation of TC Diana (1984). J. Atmos.Sci., 61, 1209-1232.

[30] Montgomery, M.T., M.E. Nicolls, T.A. Cram, and A.B. Saundess, A vortical hot tower route to tropical cyclodenesis. 2006. J.Atmos.Sci., 63, 355-386.

[31] Efimov, V.V., Shokurov M.V., Yarovaya D.A., 2007. Numerical modeling of a quasitropical cyclone over the Black Sea. Izv.-Atmos.Ocean Phys., Sea.43(6), 723-743.

[32] Montgomery M.T., Nguen V.S., Smith R.K., Persing G. 2009. Do tropical cyclones intensify by WISHE? Q.J.R, Meteorol. Soc. 135, 1697-1714.

[33] Bretherton, C.S., Blossey L.N., V.Khairoutdinov. A vortical hot tower route to tropical cyclogenesis. 2005 An energy-balance analysis of deep convective self-aggregation above uniform SST. J. Atmos. Sci. 62, 4273-4292.

[34] Nolan D.S., Rappin E.D., Emanuel K.A. 2007. Tropical cyclogenesis sensitivity to environmental parameters in radiative-convective equilibrium. Q.J.R. Meteorol. Soc. 133, 2085-2107.

[35] Nolan D.S. 2007. What is the trigger for tropical cyclogenesis? Austral. Meteorol. Mag. $56,241-266$.

[36] Jordan C.L. 1958. Mean soundings for West Indias area. J.Meteorol. 15, 91-97.

[37] Emanuel K. 2005. Increasing destructiveness of tropical cyclones over the past 30 years. Nature, 436, 685-688.

[38] Bengtsson L., Hodges K.I., Esch M., Krelyside N., Kornbluehm L., Luo J-J., Yamagata T. 2007. How may tropical cyclones change in a warmer climate? Tellus, 59A, 531-561. 
[39] Zhao M., Held I.M., Lin S-J., Vecchi G.A. 2010. Simulations of global hurricane climatology, interannual variability, and response to global warming using a $50 \mathrm{~km}$ resolution GCM.J.Climate. 22, 6653-6678. 


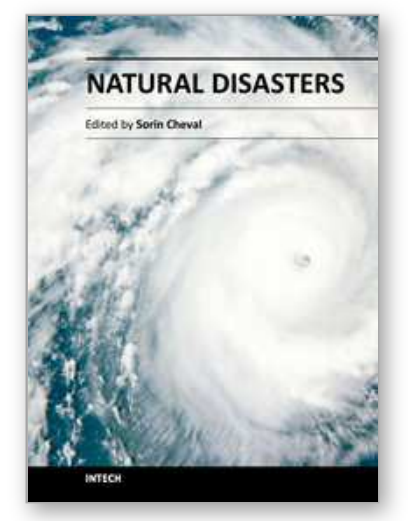

\author{
Natural Disasters \\ Edited by Dr. Sorin Cheval
}

ISBN 978-953-51-0188-8

Hard cover, 156 pages

Publisher InTech

Published online 02, March, 2012

Published in print edition March, 2012

The crossroads between a more and more populated human communities and their changing environment pose different challenges than ever before. Therefore, any attempt to identify and deliver possible solutions is more than welcome. The book Natural Disasters addresses the needs of various users, interested in a better understanding of hazards and their more efficient management. It is a scientific enterprise tackling a variety of natural hazards potentially deriving into disasters, i.e. tropical storms, avalanches, coastal floods. The case studies presented cover different geographical areas, and they comprise mechanisms for being transferred to other spots and circumstances. Hopefully, the book will be beneficial to those who invest their efforts in building communities resilient to natural disasters.

\title{
How to reference
}

In order to correctly reference this scholarly work, feel free to copy and paste the following:

G. S. Golitsyn (2012). Hurricanes: Catastrophic Effects and Their Physical Nature, Natural Disasters, Dr. Sorin Cheval (Ed.), ISBN: 978-953-51-0188-8, InTech, Available from: http://www.intechopen.com/books/naturaldisasters/hurricanes

\section{INTECH}

open science | open minds

\section{InTech Europe}

University Campus STeP Ri Slavka Krautzeka 83/A 51000 Rijeka, Croatia Phone: +385 (51) 770447

Fax: +385 (51) 686166 www.intechopen.com

\section{InTech China}

Unit 405, Office Block, Hotel Equatorial Shanghai No.65, Yan An Road (West), Shanghai, 200040, China 中国上海市延安西路65号上海国际贵都大饭店办公楼 405 单元 Phone: +86-21-62489820

Fax: +86-21-62489821 
(C) 2012 The Author(s). Licensee IntechOpen. This is an open access article distributed under the terms of the Creative Commons Attribution 3.0 License, which permits unrestricted use, distribution, and reproduction in any medium, provided the original work is properly cited. 